\title{
Radiotracer investigations to study the hydrodynamic characteristics of continuous phase in a pulsed sieve plate extraction column
}

\author{
G.U. Din ${ }^{1, \text { a }, ~ I . H . ~ K h a n ~}{ }^{1}$, I.R. Chughtai ${ }^{2}$, M.H. Inayat ${ }^{2}$ and J.H. Jin ${ }^{3}$ \\ ${ }^{1}$ Isotope Applications Division, Pakistan Institute of Nuclear Science and Technology [PINSTECH], P.O Nilore, \\ Islamabad, Pakistan \\ ${ }^{2}$ Department of Chemical Engineering, Pakistan Institute of Engineering and Applied Sciences [PIEAS], \\ P.O Nilore, Islamabad, Pakistan \\ ${ }^{3}$ Division of Physical and Chemical Sciences, Department of Nuclear Sciences and Applications, \\ International Atomic Energy Agency [IAEA], Vienna, Austria
}

\begin{abstract}
The present investigations are focused to study the hydrodynamic characteristics of continuous phase in a pulsed sieve plate extraction column using ${ }^{68} \mathrm{Ga}$ in the form of gallium chloride from an industrial radionuclide generator $\left({ }^{68} \mathrm{Ge} /{ }^{68} \mathrm{Ga}\right)$. Labeling of water with the subject radiotracer in water-kerosene environment was evaluated. Experiments for Residence Time Distribution (RTD) analysis were carried out for a range of dispersed phase superficial velocities in a liquid-liquid extraction pulsed sieve plate column operating in the emulsion regime with water as continuous and kerosene as dispersed phase. Axial Dispersion Model (ADM) was used to simulate the hydrodynamic characteristics of continuous phase. It has been observed that the axial mixing in the continuous phase decreases and slip velocity increases with increase in superficial velocity of dispersed phase while the holdup of continuous phase was found to decrease with increase in superficial velocity of dispersed phase. ADM with open-open boundary condition was found to be a suitable model for the subject system.
\end{abstract}

\section{INTRODUCTION}

The concept of pulsed liquid-liquid extraction column is attributed to Van Dijck, 1935 [1]. This apparatus is very efficient as it offers large interfacial area, high mass transfer coefficient, high turbulence and minimum radial gradients. Phases in this kind of equipment are subject to flow counter currently to achieve high concentration gradients for efficient mass transfer but axial mixing in both phases lowers the process efficiency by lowering solute concentration gradients. As the major sources of axial mixing are geometrical and operating parameters, therefore, its presence is inevitable and needs special care. A usual process engineering approach to represent the hydrodynamics of phases in this kind of extractors is a plug flow model with some degree of back mixing superimposed on it $[2,3]$ and the concept of Residence Time Distribution (RTD) analysis is an important tool for the estimation of axial mixing [4]. The holdup and slip velocity of phases are other important parameters in the design and operation of pulsed extraction columns.

The issue of axial mixing in the continuous phase of pulsed liquid-liquid extraction columns has been under consideration of various researchers. Effect of various geometrical and operating parameters on the axial mixing of continuous phase has been reported in these investigations [5-9]. Most of these studies have been carried out using visual method after injecting a dye tracer or a conventional method by injecting a non-radioactive tracer and measurements were made by a conductivity probe. These experimental approaches

\footnotetext{
a e-mail: ghiyas@pinstech.org.pk; ghiyasuddin@hotmail. com (Ghiyas Ud Din)
}

present some disadvantages including low sensitivity and poor statistics.

Radiotracer technology offers state of the art technique for industrial process optimization and trouble shooting due to high sensitivity, on-line measurement, better statistics and high benefit to cost ratio. The unique ability of this technology is that it can provide information that may not be obtained by other techniques. This technology has been widely used in developed countries to help solve industrial problems but still underutilized in developing countries due to the unavailability of radiotracers at the time of requirement. For developing countries that do not possess radioisotope production facilities, it is necessary to import the radiotracers and long time involved in this process rules out the possibility of achieving potential benefit of this technology. Medical radionuclide generators such as ${ }^{99} \mathrm{Mo} /{ }^{99 \mathrm{~m}} \mathrm{Tc}$ and ${ }^{113} \mathrm{Sn} /{ }^{113 \mathrm{~m}} \mathrm{In}$ provide a partial solution to the problem but radiotracers from these generators have limited applications in industry because of their relatively short half lives, low gamma energies and adsorption to solid substances depending on the chemical and physical conditions. Hence, there is a need to explore some more nuclear genetic relationships that may form the basis for the development of radionuclide generators for industrial process investigations. Keeping in view of these considerations, the IAEA has developed a new industrial radionuclide generator (IRG) system $\left({ }^{68} \mathrm{Ge} /{ }^{68} \mathrm{Ga}\right.$ ) especially for industrial process investigations [10]. The concept of ${ }^{68} \mathrm{Ge} /{ }^{68} \mathrm{Ga}$ generator is already in the market for nuclear medicine applications especially for Positron Emission Tomography [11-14] but it is rather new in the field of industrial process investigations.

The present investigations are focused to study the hydrodynamics of continuous phase (water) in a pulsed sieve plate extraction column using radiotracer 
technique. The suitability of ${ }^{68} \mathrm{Ga}$ in the form of gallium chloride as water tracer in water-kerosene environment was tested before injecting it into the system. RTD experiments were carried out for a wide range of dispersed phase superficial velocities to study the axial mixing of continuous phase. Holdup of continuous phase and slip velocity are also reported. Hydrodynamics of the system was modeled using the Axial Dispersion Model (ADM) with open-open boundary condition and the results are discussed.

\section{MATERIALS AND METHODS}

\subsection{Experimental}

${ }^{68} \mathrm{Ge} /{ }^{68} \mathrm{Ga}$ radionuclide generator-based radiotracer is used in presented investigations. This radionuclide generator is comprised of carrier-free germanium $\left({ }^{68} \mathrm{Ge}\right)$ absorbed on a tin dioxide column. The special feature of this IRG is that it has been made cost economical by compromising the biological factors, therefore, it is not useful for in-vivo applications. This IRG system produces ${ }^{68} \mathrm{Ga}$ radiotracer in the form of gallium chloride with dual gamma energy $0.511 \mathrm{MeV}$. The beauty of this IRG lies in the half-lives of mother (270 days) and daughter (67.6 minutes). So the life of this IRG spans over a period of around two years and due to high gamma energy of the daughter it is useful in industrial systems having thick metallic walls. Moreover, the short half-life of daughter provides the possibility of quick decontamination of the systems under investigation. Although ${ }^{99 \mathrm{~m}} \mathrm{Tc}$ in the form of sodium pertechnatate proved to be a good water tracer while working on the hydrodynamics of dispersed phase (water) in the pulsed sieve plate extraction column [15] but this radiotracer failed to provide a sufficient signal at the system outlet due to large degree of dilution as the water is subject to flow as continuous phase in the present scenario. However, thick lead shield collimators with fine collimation are required to counter high back ground level, which may arise during the course of experiments.

In order to test the suitability of ${ }^{68} \mathrm{Ga}$ in the form of gallium chloride as water tracer in water-kerosene environment, a minute quantity of radiotracer was mixed thoroughly in equal amounts of water and kerosene in a glass beaker with the help of a stainless steel stirrer. The constituents were allowed to separate under gravity and the level of radioactivity was measured with the help of a collimated $\mathrm{NaI}(\mathrm{Tl})\left(2^{\prime \prime} \times 2^{\prime \prime}\right)$ detector placed in a fix geometry. Upon measurements, the radiotracer was found suitable as water tracer in water- kerosene environment as no activity was found in the organic phase (kerosene). Moreover, no adsorption of radiotracer was experienced on the glass wall and stainless steel stirrer upon rinsing them with fresh water and kerosene.

The schematic diagram of pulsed sieve plate extraction column under investigation is shown in Figure 1. The internal diameter of the column is $5 \times 10^{-2} \mathrm{~m}$ and height is $2 \mathrm{~m}$. Two separating chambers, one at the top and the other at the bottom of the column are also part of this apparatus. The column is fitted with regularly
Table 1. Column specifications and operating parameters.

\begin{tabular}{ll}
\hline Internal diameter of the column $(\mathrm{m})$ & $5 \times 10^{-2}$ \\
Length of the column $(\mathrm{m})$ & 2 \\
Number of sieve plates & 38 \\
Plate spacing $(\mathrm{m})$ & $5 \times 10^{-2}$ \\
Plate diameter $(\mathrm{m})$ & $5 \times 10^{-2}$ \\
Hole size $(\mathrm{m})$ & $2 \times 10^{-3}$ \\
Average number of holes & 140 per plate \\
Average free area $(\%)$ & 25 \\
Pulsation frequency $\left(\mathrm{s}^{-1}\right)$ & 1.56 \\
Pulsation amplitude $(\mathrm{m})$ & $1 \times 10^{-2}$ \\
Continuous phase superficial & $0.47 \times 10^{-2}$ \\
velocity $(\mathrm{m} / \mathrm{s})$ & \\
Range of dispersed & $0.25 \times 10^{-2}-0.5 \times 10^{-2}$ \\
phase superficial velocity $(\mathrm{m} / \mathrm{s})$ & \\
\hline
\end{tabular}

spaced $\left(5 \times 10^{-2} \mathrm{~m}\right)$ sieve plates, which help to increase the interfacial area between the two immiscible liquids. The column was operated counter currently with heavy phase (water) as continuous and light phase (kerosene) as dispersed phase. The kerosene which is fed into the lower separating chamber with the help of a metering pump flows upwards through the sieve plate column to the upper separating chamber where it overflows to a collection vessel. Similarly water is fed into the top separating chamber via a metering pump from where it flows downwards through the column to the lower separating chamber, and through a balance leg into a collection vessel. A pulse unit located at the base of lower separating chamber provides vertical pulses to the flowing fluids. The column was operated in the emulsion regime i.e. dispersed phase remained dispersed throughout the plate stack and no coalescence into layers occurred at the plates. A liquid-liquid interface was allowed to form at about $10 \mathrm{~cm}$ above the heavy phase inlet and this interface level was stabilized with the help of a balance leg before starting an experiment.

About $0.25 \mathrm{mCi}$ of ${ }^{68} \mathrm{Ga}$ eluted from a ${ }^{68} \mathrm{Ge} /{ }^{68} \mathrm{Ga}$ generator was injected in the form of an instantaneous pulse to carry out RTD experiments for investigation of the hydrodynamics of continuous phase (water) as per experimental plan shown in Figure 1. The experiments were carried out for a range of dispersed phase superficial velocities as given in Table 1 . The movement of radiotracer was monitored for every second with the help of lead collimated $\mathrm{NaI}(\mathrm{Tl})\left(2^{\prime \prime} \times 2^{\prime \prime}\right)$ scintillation detectors mounted at $D_{1}, D_{2}$ and $D_{3}$ as shown in Figure 1 . The data was acquired on-line using a multi-channel data acquisition system and stored in a computer for processing.

\subsection{Data analysis}

The tracer data from detectors $\mathrm{D}_{2}$ (column inlet) and $\mathrm{D}_{3}$ (column outlet) was corrected for background, radioactive decay and normalized. The experimental Mean Residence Time (MRT) of the system was calculated by the difference of first moments of outlet and inlet response curves. 


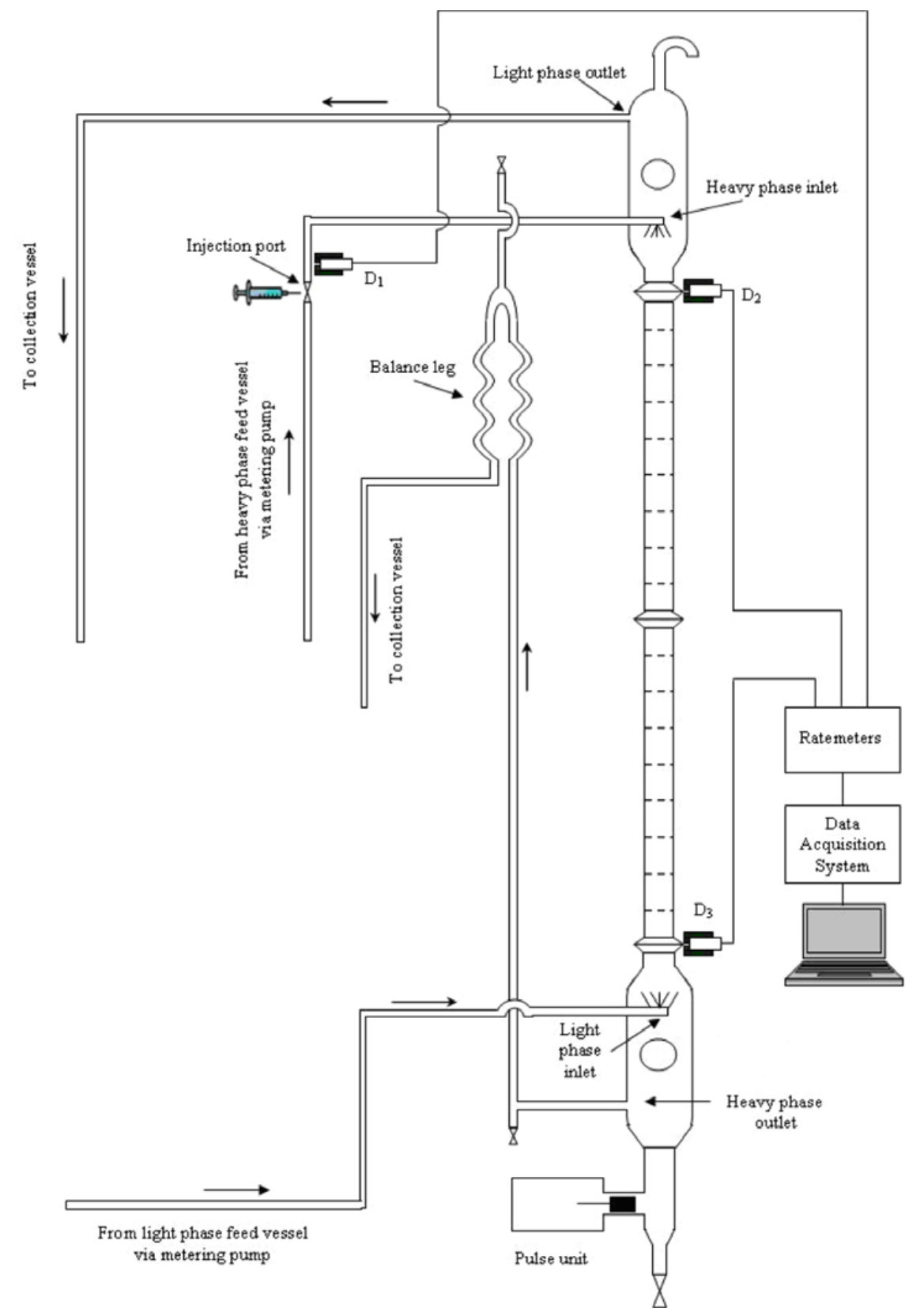

Figure 1. Schematic diagram of pulsed sieve plate extraction column.

Mathematical expression for the first moment in discrete form can be written as:

$$
\text { First Moment }=\frac{\sum_{i} t_{i} C_{i} \Delta \mathrm{t}_{\mathrm{i}}}{\sum_{i} C_{i} \Delta t_{i}} .
$$

\section{Where}

$$
C=\text { Tracer concentration (counts } / \text { in present case) }
$$

$t=$ Time of measurement $(s)$

$\Delta t=$ Time interval between the two measurements $(s)$ $i=0,1,2,3, \ldots \ldots \ldots \ldots \ldots$

Overall holdup of the phase under investigation was calculated on the basis of calculated MRT using the following relationship:

$$
H_{c}=\frac{\bar{t} Q_{c}}{V_{R}}
$$

Where

$$
\begin{aligned}
H_{c} & =\text { Continuous phase holdup } \\
\bar{t} & =\text { Mean residence time } \\
Q_{c} & =\text { Continuous phase flow rate } \\
V_{R} & =\text { Effective reactor volume. }
\end{aligned}
$$

The slip velocity of continuous phase averaged over the whole column was estimated from the above calculated $H_{c}$ using the following equation [16]:

$$
V_{s}=\frac{U_{c}}{H_{c}}+\frac{U_{d}}{\left(1-H_{c}\right)} .
$$


Where

$$
\begin{aligned}
V_{s} & =\text { Slip velocity of dispersed phase } \\
U_{c} & =\text { Continuous phase superficial velocity } \\
U_{d} & =\text { Dispersed phase superficial velocity. }
\end{aligned}
$$

The Residence Time Distribution (RTD) is a probability distribution function that describes the amount of time a fluid element spends inside a reactor. It helps in troubleshooting of reactors and characterizes the mixing and flow within the reactors. If an impulse of tracer is injected at the inlet of a system at time $t=0$ and its concentration is measured as a function of time at the outlet, then $E(t)$ representing the probability for a tracer element to have a residence time between the time interval $(t, t+d t)$ is defined as:

$$
E_{i}(t)=\frac{C_{i}(t)}{\int_{0}^{\infty} C_{i}(t) d t} .
$$

Such that

$$
\int_{0}^{\infty} E_{i}(t) d t=1
$$

Where

$$
\begin{aligned}
& i=1,2,3, \ldots \ldots \ldots \ldots, n \\
& C_{i}(t)=\text { Tracer concentration } \\
& E_{i}(t)=\text { Residence Time Distribution function } .
\end{aligned}
$$

RTD models have been playing a vital role for industrial process investigations for decades. They provide macroscopic lumped sum description, which is sufficient for many engineering calculations. The plug flow is an ideal condition for the flow of phases in an extraction column but some degree of axial mixing is always inevitable. Axial Dispersion Model (ADM) was used to study the system hydrodynamics. The flow conditions are not plug type before and after the inlet $\left(\mathrm{D}_{2}\right)$ and outlet $\left(D_{3}\right)$ boundaries, therefore, open-open boundary condition can be chosen in present situation. A uniform radial concentration in the continuous phase is assumed due to large length to diameter ratio.

The basic general differential equation of the one dimensional ADM for fluid flow in the dimensionless form is as follows:

$$
\frac{\partial C}{\partial \theta}=\frac{1}{P e} \frac{\partial^{2} C}{\partial X^{2}}-\frac{\partial C}{\partial X}
$$

Where

$$
\begin{aligned}
C & =\text { Dimensionless tracer concentration }=\frac{c(t)}{c(0)} \\
P e & =\text { Peclet number }=\frac{U_{c} L}{D} \\
X & =\text { Dimensionless axial coordinate }=\frac{x}{L} \\
D & =\text { Axial dispersion coefficient } \\
c(t) & =\text { Tracer concentration at time } t \\
c(0) & =\text { Initial tracer concentration. }
\end{aligned}
$$

A solution of Eq. (6) for open-open boundary condition in dimensionless form is given as under with a detailed

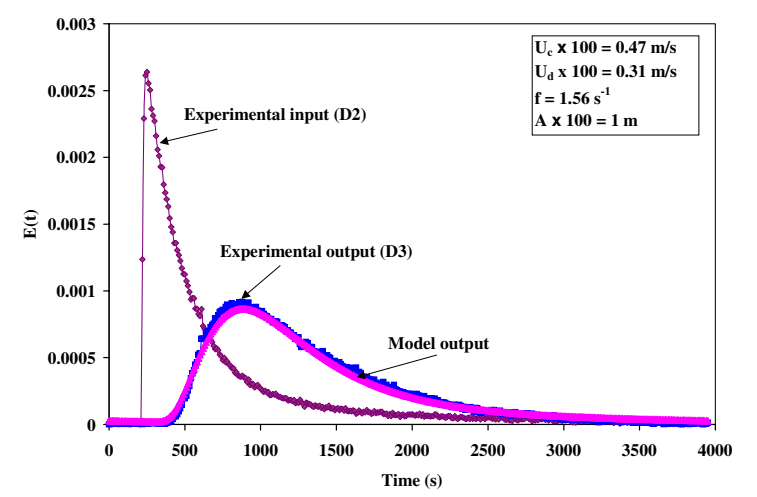

Figure 2. Typical normalized RTD function curves with model output response of continuous phase.

analysis given by $[2,3]$ :

$$
E(\theta)=\sqrt{\frac{P e}{4 \pi \theta}} \exp \left(\frac{-P e(1-\theta)^{2}}{4 \theta}\right) .
$$

A Residence Time Distribution analysis software package "RTD" developed by IAEA [17] was used for modeling in the present investigations. Axial Dispersion Model (ADM) with two points measurement methodology in this software package was used. It optimizes two parameters, the MRT and Pe. This model calculates the RTD response of a system to an arbitrary pulse of tracer by convoluting the input function with impulse response of the model [3-17]. It uses the least square curve fitting method to fit the model RTD function (Eq. (7)) onto the experimental data and obtains the optimum model parameters. Figure 2 shows typical normalized RTD function curves obtained at the input $\left(D_{2}\right)$ and output $\left(D_{3}\right)$ with model output response of continuous phase in response to an instantaneous pulse injection at $\left(\mathrm{D}_{1}\right)$.

Due to the random nature of radioactive decay process, any measurement of radiation is subject to some degree of statistical fluctuation. These inherent fluctuations represent an unavoidable source of uncertainty in all nuclear measurements and are a predominant source of error in present investigations.

Error associated in the measurement of radiation and its propagation in subsequent calculations has been worked out using standard methods and shown as error bars in respective results $[18,19]$. The metering pumps used for the flow of fluids and pulsation in the pulsed sieve plate column were calibrated before the experiments and errors associated in the measurement of flow rate, pulsation frequency and amplitude have been considered negligible.

\section{RESULTS AND DISCUSSION}

Figure 3(a-d) shows the effect of $U_{d}$ on the MRT, holdup, slip velocity and axial mixing in the continuous phase when $U_{c}$, pulsation frequency and amplitude are kept constant. It has been observed that increase in $U_{d}$ decreases the MRT of the continuous phase. As $U_{d}$ increases, the droplet population density of dispersed phase inside the column increases; hence increase the dispersed phase 

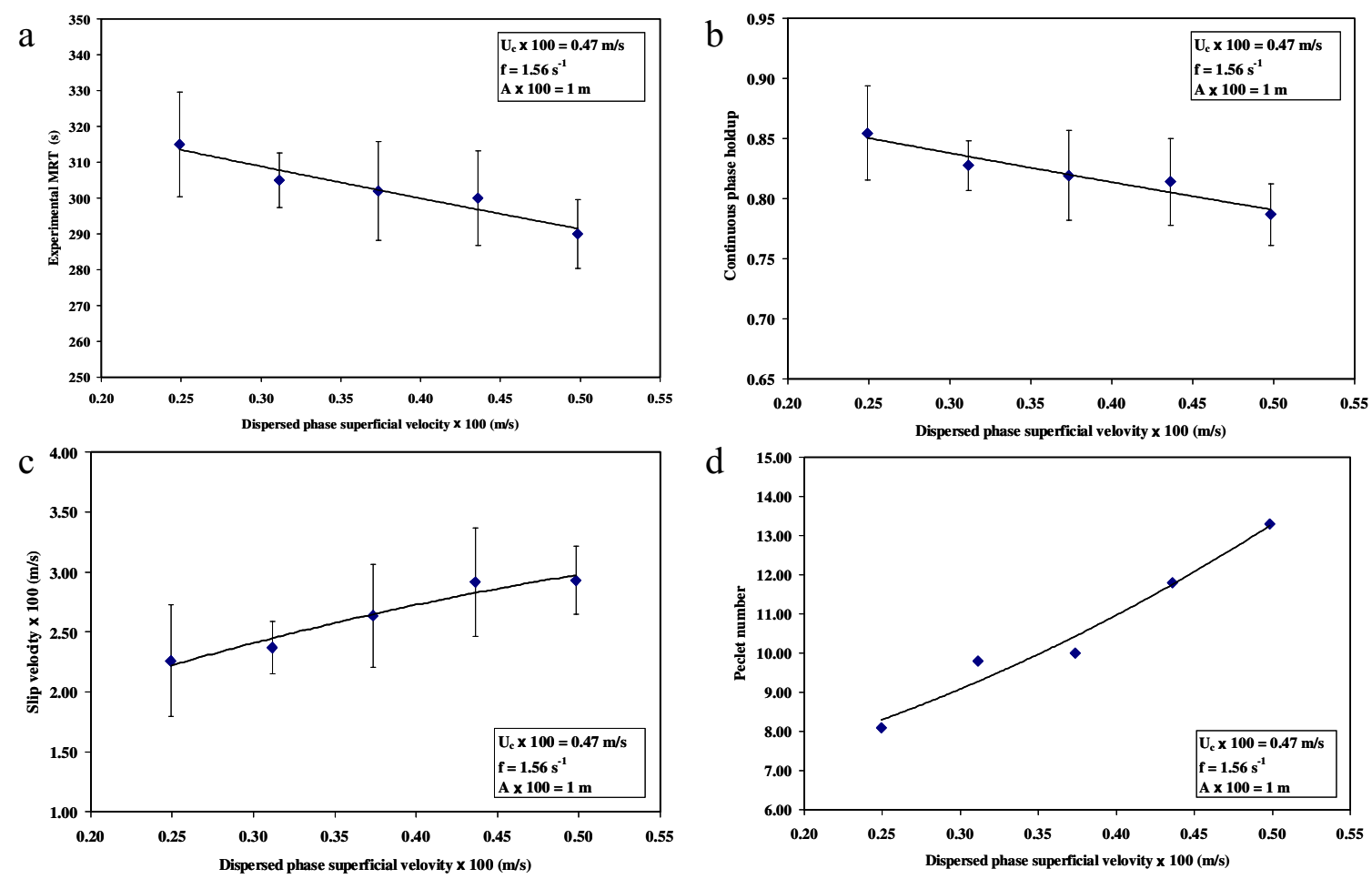

Figure 3. Effect of dispersed phase superficial velocity on the (a) MRT of continuous phase (b) Holdup of continuous phase (c) Slip velocity (d) Pe Number of continuous phase.

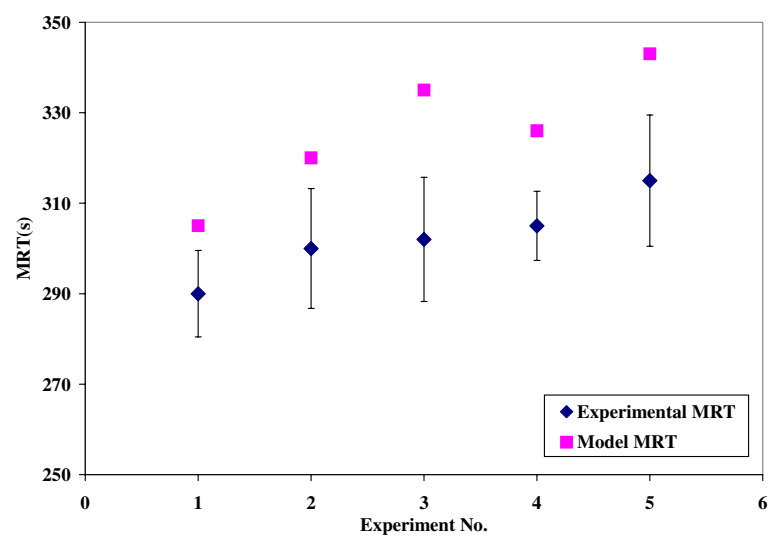

Figure 4. Comparison of experimental and model MRTs.

holdup. The increase in holdup of dispersed phase with increase in $U_{d}$ has already been reported while working on the hydrodynamics of dispersed phase on the same column with water as dispersed and kerosene as continuous phase [15]. The increase in dispersed phase holdup decreases the proportion of continuous phase inside the column (Fig. 3b) leading to a decrease in the MRT of continuous phase (Eq. (2)). As the flow rate of dispersed phase increases, the slip velocity increases as shown in Figure $3 \mathrm{c}$. An increase in advection in the continuous phase leads to decrease in axial mixing of continuous phase. This phenomenon can be seen by the increasing Peclet number (Fig. 3d).

A comparison of experimental and model MRTs of various RTD experiments carried out during these investigations has been given in Figure 4 which shows a good agreement between experimental and model MRTs.

\section{CONCLUSIONS}

(a) ${ }^{68} \mathrm{Ga}$ in the form of gallium chloride is a suitable radiotracer for labeling water phase in waterkerosene environment.

(b) The axial mixing in the continuous phase of a liquid-liquid extraction pulsed sieve plate column decreases with increase in superficial velocity of dispersed phase.

(c) The holdup of continuous phase decreases with increase in superficial velocity of dispersed phase.

(d) The slip velocity increases with increase in superficial velocity of dispersed phase.

(e) Axial Dispersion Model (ADM) is a suitable model to describe the hydrodynamics of continuous phase in pulsed sieve plate extractions column.

The authors are grateful to the Higher Education Commission [HEC] for financial support in accomplishment of this study. The authors are greatly indebted to the International Atomic Energy Agency (IAEA) for partially supporting this research under the framework of its Coordinated Research Project (F.2.20.44) on "Evaluation and Validation of Radionucilde Generator-based Radiotracers for Industrial Applications" and for the provision of RTD analysis software package. The cooperation and technical assistance extended by Pakistan Institute of Nuclear Science and Technology [PINSTECH] and Pakistan Institute of Engineering and Applied Sciences [PIEAS] is thankfully acknowledged. 


\section{References}

[1] W.J.D. Van Dijck, U.S. Pat. No. 2011186 (1935)

[2] O. Levenspiel, W.K. Smith, Chem. Eng. Sci. 6 (4-5), 227-235 (1957)

[3] O. Levenspiel, Chemical reaction engineering, John Wiley \& Sons, New York (1999)

[4] P.V. Danckwerts, Chem. Eng. Sci. 2 (1), 1-13 (1953)

[5] S.D. Kim, M.H.I. Baird, Can. J. Chem. Eng. 54, 81-89 (1976a)

[6] S.D. Kim, M.H.I. Baird, Can. J. Chem. Eng. 54, 235-237 (1976b)

[7] M.M. Hafez, M.H.I. Baird, I. Nirdosh, Can. J. Chem. Eng. 57, 150-158 (1979)

[8] P. Parthasarathy, G. Sriniketan, N.S. Srinivas, Y.B.G. Varma, Chem. Eng. Sci. 39 (6), 987-995 (1984)

[9] G. Srinikethan, A. Prabhakar, Y.B.G. Varma, Bioprocess Eng. 2 (4), 161-168 (1987)

[10] International Atomic Energy Agency, Report of the first research coordination meeting of the CRP on evaluation and validation of radioisotope generators-based radiotracers for industrial applications. IAEA, Vienna, Austria (2007)
[11] K. Casteleyn, R. Franceschini, F. Lunghi, G. Macchi, L. Manes, E. Seccamani, B. Weckermann, Appl. Radiat. Isot. 45 (3), 395-396 (1994)

[12] M. Nakayama, M. Haratake, M. Ono, T. Koiso, K. Harada, H. Nakayama, S. Yahara, Y. Ohmomo, Y. Arano, Appl. Radiat. Isot. 58 (1), 9-14 (2003)

[13] C.J. Mathias, M.A. Green, Appl. Radiat. Isot. doi:10.1016/j.apradiso.2008.06.004 (2008)

[14] T.H. Bokhari, M. Ahmad, I.U. Khan, Appl. Radiat. Isot. doi:10.1016/j.apradiso.2008.08.004 (2008)

[15] G.U. Din, I.R. Chughtai, M.H. Inayat, I.H. Khan, Appl. Radiat. Isot. 66, 1818-1824 (2008)

[16] D. Venkatanarasaiah, Y.B.G. Varma, Bioprocess Eng. 18(1), 119-126 (1998)

[17] International Atomic Energy Agency, Integration of tracing with computational fluid dynamics for industrial process investigation, Tecdoc-1412. IAEA, Vienna, Austria (2004)

[18] G.F. Knoll, Radiation detection and measurement, John Wiley \& Sons, New York (2000)

[19] P.R. Bevington, Data reduction and error analysis for the physical sciences, Mc Graw Hill Co. Inc., New York (2003) 